\title{
AVALIAÇÃO DA SEVERIDADE DAS MANCHAS DE Phaeosphaeria E DE Cercospora DO MILHO EM CULTIVO DE SAFRINHA $^{1}$
}

\author{
Gisèle Maria Fantin ${ }^{2}$ \\ Christina Dudienas ${ }^{3}$ \\ Jairo Lopes de Castro ${ }^{4}$ \\ Eduardo Sawazaki ${ }^{3}$ \\ Aildson Pereira Duarte ${ }^{5}$
}

\section{RESUMO}

As manchas foliares de Phaeosphaeria e de Cercospora apresentam elevada importância à cultura do milho no Brasil, sendo muito freqüentes no Sul do Estado de São Paulo nos cultivos de outono-inverno. Este trabalho teve como objetivo comparar épocas de avaliação e métodos de quantificação destas doenças do milho, em cultivo de safrinha, verificando sua influência na produtividade. Foi instalado um ensaio em Capão Bonito-SP, em 2002, com 42 híbridos simples ou triplos comerciais de milho, em 3 blocos ao acaso. Avaliações da severidade das doenças foram realizadas aos 83, 92, 106 e 115 dias após a semeadura (d.a.s.), utilizando uma escala de notas de 1 a 9 , correspondentes a $0 ; 1 ; 2,5 ; 5$; $10 ; 25 ; 50 ; 75$ e mais de $75 \%$ de área foliar com sintomas. Obteve-se também a área abaixo da curva de progresso da doença (AACPD) para cada uma delas e a soma de ambas. As duas doenças ocorreram com elevada severidade. Os coeficientes das correlações fenotípicas (r) da

$\overline{1 . ~ T r a b a l h o ~ a p r e s e n t a d o ~ d u r a n t e ~ o ~} 7^{\circ}$ Seminário Nacional de Milho Safrinha, Rio Verde, 2003.

2. Instituto Biológico (IB), Caixa Postal 70, CEP 13001-970, Campinas-SP, e-mail: gisele(a) biologico.sp.gov.br

3. Instituto Agronômico (IAC), Caixa Postal 28, CEP 13001-970, Campinas-SP.

4. APTA Regional Sudoeste Paulista, Caixa Postal 62, CEP 18300-970, Capão Bonito-SP.

5. APTA Regional Médio Paranapanema, Caixa Postal 263, CEP 19800-000, Assis-SP. 
severidade das doenças nas quatro épocas e da AACPD com a produtividade foram negativos e altamente significativos $(\mathrm{P}<0,001$, o que indicaremos por 3 asteriscos $* * *)$. O coeficiente de correlação da AACPD total com a produtividade foi $-0,76^{* * *}$. As melhores correlações entre cada uma das doenças e as produtividade foram obtidas na quarta época de avaliação para a mancha de Phaeosphaeria $\left(\mathrm{r}=-0,70^{* * *}\right)$ e na terceira e quarta para a mancha de Cercospora $(\mathrm{r}=-0,61 * * *$ e $-0,59 * * *$, respectivamente), quando as plantas ainda se encontravam no final do estádio de grão pastoso, devido ao alongamento do ciclo do milho que ocorre durante o outono-inverno. As AACPDs de cada uma das doenças apresentaram coeficientes de correlação com a produtividade semelhantes aos das avaliações tardias, mostrando-se também adequadas para demonstrar a influência das doenças na produtividade $(-0,69 * * *$ e $-0,61 * * *$ para as manchas de Phaeosphaeria e de Cercospora, respectivamente). Os híbridos mais resistentes à mancha de Phaeosphaeria foram A 2560, Fort e Strike seguidos por AG 7575, 30K75, XGN 01101 e Valent è mancha de Cercospora foram A 2560, Strike, DKB 333B, A 2555 e XB 7012. Os híbridos mais produtivos, sob a ocorrência destas doenças, foram DKB 333B e A 2560, seguidos por Fort, Valent e 30F90 e estes por 30K75, Strike, DAS 8480, DKB 350, Tork e XB 7012. Alguns híbridos, como 30F90 e DAS 8480, tiveram elevada produtividade, mesmo apresentando moderada porcentagem de área foliar coberta por manchas. A influência das doenças na produtividade média dos híbridos foi estimada procurando minimizar o efeito das diferenças de potencial produtivo entre eles, agrupando-os, com base no teste de Scott-Knott $(\mathrm{P}<0,05)$, em seis classes de AACPD total. Obteve-se, desta forma, $\mathrm{r}=-0,94 * * *$ da AACPD total com a produtividade e observaram-se decréscimos na produtividade dos híbridos a partir de níveis de severidade inferiores a $2,5 \%$ de área foliar com sintomas de cada uma das doenças, chegando a reduções médias de produtividade próximas a $2.000 \mathrm{~kg} / \mathrm{ha}$, com severidade ao redor de $25 \%$ e $10 \%$ de área foliar com sintomas das manchas de Phaeosphaeria e de Cercospora, respectivamente. Concluiu-se, neste trabalho, que uma avaliação no final do estádio de grão pastoso foi suficiente para discriminar a resistência dos híbridos a ambas as doenças. Também se evidenciou a importância das doenças mesmo em baixos niveis de severidade. 
Palavras-chave: Mancha foliar, Zea mays, Phaeosphaeria maydis, Cercospora zeae-maydis, produtividade, segunda safra.

\section{ABSTRACT}

\section{EVALUATION OF Phaeosphaeria LEAF SPOT AND GRAY LEAF SPOT SEVERITY OF MAIZE IN THE AUTUMN-WINTER CROP}

Phaeosphaeria leaf spot and gray leaf spot are highly important maize diseases in Brazil, being very frequent in the South of São Paulo State in the off-season crop. This work had the objective to compare periods of evaluation and quantification methods of these diseases in maize, in the autumn-winter crop, and to verify its influence on the yield. A trial was carried out in Capão Bonito-SP, in 2002, with 42 simple or triple commercial maize hybrids in 3 randomized blocks. Evaluations of disease severity were accomplished at $83,92,106$ and 115 days after sowing (d.a.s.), using a scale of grades from 1 to 9 , corresponding to 0 ; $2.5 ; 5 ; 10 ; 25 ; 50 ; 75$ and more than $75 \%$ of the foliar area with disease symptoms. The area under the disease progress curve (AUDPC) for each disease and total were also calculated. Both diseases occurred with high severity. Negative and highly significant $(\mathrm{P}<0.001)$ phenotypic correlation coefficients ( $r$ ) of the disease severity at the four dates and of AUDPC with the yield were observed. The correlation coefficient of total AUDPC with the yield was $-0,76^{* * *}$. The best correlations between each one of the diseases and yield were obtained at the fourth evaluation date for the Phaeosphaeria leaf spot $\left(\mathrm{r}=-0.70^{* *}\right)$ and at the third and fourth time, for the gray leaf spot $\left(\mathrm{r}=-0.61^{* * *}\right.$ and $-0.59^{* * *}$, respectively), when the plants were still at the end of dough stage, due to the prolongation of the cycle of the plants that takes place in the autumn-winter conditions. The correlation coefficient between AUDPC of each disease and yield ($0.69^{* * *}$ e $-0.61^{* * *}$ to Phaeosphaeria leaf spot and gray leaf spot, respectively) were close to that of the last evaluations, showing to be also appropriate to demonstrate the influence of the diseases on the yield. The most resistant hybrids to Phaeosphaeria leaf spot were A 2560, Fort 
and Strike followed by AG 7575, 30K75, XGN 01101, Valent, DKB 333B and DKB 350 and the most resistant to gray leaf spot were A 2560, Strike, $\mathrm{DKB} 333 \mathrm{~B}, \mathrm{~A} 2555$ and $\mathrm{XB} 7012$. The high yielding hybrids under these diseases were DKB 333B and A 2560 followed by Fort, Valent and 30F90 and these by $30 \mathrm{~K} 75$, Strike, DAS 8480, DKB 350, Tork and XB 7012. Some hybrids, as 30F90 and DAS 8480 , showed high yield, even with moderate percentage of foliar area covered by spots. The influence of the diseases on the medium yield of the hybrids was estimated by minimizing the effect of the differences of productive potential among the hybrids, being these grouped, using the test of Scott-Knott $(\mathrm{P}<0.05)$, in six classes of total AUDPC. The coefficient obtained was $\mathrm{r}=-0.94 * * *(\mathrm{P}<0.001)$ of this medium AUDPC with the yield. The yield decreased even for severity levels lower than 2,5\% of foliar area affected by each disease. Decreases close to $2.000 \mathrm{~kg} / \mathrm{ha}$ on the yield were observed under severity of approximately $25 \%$ and $10 \%$ of foliar area with Phaeosphaeria leaf spot and gray leaf spot symptoms, respectively. This study indicated that the disease evaluation in one period, at the end of dough stage, was enough to differentiate the resistance of the hybrids to both diseases, and showed their importance even at low levels of disease severity.

Key words: Leaf spot, Zea mays, Phaeosphaeria maydis, Cercospora zeae-maydis, yield, off-season crop.

\section{INTRODUÇÃO}

Entre as doenças que apresentam maior importância à cultura do milho (Zea mays L.), no Brasil estão as manchas foliares de Phaeosphaeria e de Cercospora. A primeira, causada por Phaeosphaeria maydis (P. Henn.) Rane, Payak \& Renfro com anamorfa Phoma sp. (White, 2000), começou a se evidenciar no início da década de 90 (Fantin, 1994) e apresenta distribuição generalizada nas áreas produtoras de milho do País, tanto na safra como na safrinha. Danos à produtividade causados por esta doença foram estudados por vários autores (Menten et al., 1996; Sawazaki et al., 1997; Brasil \& Carvalho, 1998; Pacheco et al., 1999; Fantin et al., 2000; Pegoraro et al., 2001 e Von Pinho et al., 2002). 
A mancha de Cercospora, também denominada cercosporiose, pode ser causada por duas espécies de Cercospora: C. zeae-maydis Tehon \& E. Y. Daniels com teleomorfa Mycosphaerella sp. e C. sorghi var. maydis Ell. \& Ev. (Shurtleff, 1992). Epidemias da doença são atribuídas principalmente à primeira espécie. A cercosporiose, embora mundialmente relevante há mais de duas décadas (Ward et al., 1999, White, 2000), adquiriu maior importância no Brasil no final da década de 90, causando epidemias no Centro-Oeste do País na safra 1999/2000 (Fernandes \& Oliveira, 2000). Foi relatada já ocorrendo com alta intensidade no Estado de São Paulo na safra seguinte (Fantin et al., 2001) e vem-se evidenciando desde então (Fantin et al., 2004a). Diferenças quanto à resistência dos híbridos a esta doença são citadas por Fantin et al. (2001 e 2004b).

Tanto a mancha de Phaeosphaeria como a de Cercospora são muito favorecidas por umidade elevada, condição que ocorre com bastante freqüência ao sul do Estado de São Paulo. Por outro lado, MiguelWruck et al. (2002) observaram, em ensaios instalados em várias regiões do Estado de Minas Gerais, que o manejo da cultura em uma região (plantios subseqüentes de milho de verão, de inverno e irrigado, muitas vezes sem rotação de culturas) teve maior influência do que o ambiente na severidade dessas doenças.

Devido à elevada importância das manchas de Phaeosphaeria e de Cercospora para a cultura do milho, e ao pouco conhecimento sobre esta segunda doença em nosso País, este trabalho teve como objetivo comparar épocas de avaliação e métodos para quantificação da severidade destas doenças e ainda verificar seu efeito sobre a produtividade do milho, em híbridos comercializados atualmente, com diversos graus de resistência.

\section{MATERIAL E MÉTODOS}

Foi instalado um experimento em Capão Bonito, na região Sul do Estado de São Paulo, em 12/03/02, como parte de um grupo de ensaios de avaliação regional de cultivares. Os tratamentos consistiram de 42 cultivares de milho (híbridos simples ou triplos), sendo utilizados três blocos ao acaso. As parcelas foram constituídas de quatro linhas de $5 \mathrm{~m}$, espaçadas de $90 \mathrm{~cm}$, sendo as duas centrais consideradas como parcela 
útil. A adubação no plantio foi de $250 \mathrm{~kg} \cdot \mathrm{ha}^{-1}$ da fórmula NPK 8-28-16 e a de cobertura $60 \mathrm{~kg} \cdot \mathrm{ha}^{-1}$ de nitrogênio da fonte sulfato de amônio. $\mathrm{O}$ florescimento masculino ocorreu de 57 a 69 dias após a semeadura, isto é, 63 dias em média.

Avaliações da severidade das manchas foliares de ocorrência natural foram realizadas em 4 épocas, aos 83, 92, 106 e 115 dias após a semeadura, atribuindo-se, por parcela, notas de 1 a 9, correspondendo aos níveis de $0 ; 1 ; 2,5 ; 5 ; 10 ; 25 ; 50 ; 75$ e mais de $75 \%$ de área foliar afetada, com auxílio de uma escala diagramática elaborada pela Agroceres (Agroceres, 1993). Calculou-se também a área abaixo da curva de progresso da doença (AACPD), para as duas doenças, através da fórmula: AACPD $=\sum\left[\left(\left(Y_{i}+Y_{i+1}\right) / 2\right) *\left(T_{i+1}-T_{i}\right)\right]$ onde $Y_{i}=$ severidade da doença na época de avaliação $i ; Y_{i+1}=$ severidade da doença na época de avaliação $\mathrm{i}+1 ; \mathrm{T}_{\mathrm{i}}=$ época da avaliação $\mathrm{i}$ em número de dias após a semeadura; $\mathrm{T}_{i+1}=$ época da avaliação $i+1$. A colheita foi realizada em 28/08/02.

Obteve-se a produtividade de grãos (em $\mathrm{kg} / \mathrm{ha}$ ) corrigindo-se a umidade para $13 \%$. Foi realizada análise de variância das notas de severidade nas quatro épocas, da AACPD de cada uma das doenças, da AACPD da soma de ambas (total) e da produtividade. Obteve-se o coeficiente de correlação fenotípica (r) da severidade das doenças em cada época e das AACPDs com a produtividade. A comparação de médias foi feita pelo teste de Scott-Knott a 5\% através do programa SASM-Agri (Canteri et al., 2001). Para análise, os dados (x) de severidade de cada uma das doenças foram transformados em $\sqrt{x+1}$.

Para o estudo da influência das doenças sobre a produtividade média do milho, procurou-se minimizar o efeito das diferenças de potencial produtivo de cada cultivar, agrupando-as em classes de resistência, com base na AACPD de cada uma das doenças e total, com o teste de Scott-Knott a $5 \%$. Foram obtidas as médias de severidade e de produtividade em cada classe. Obteve-se o coeficiente de correlação fenotípica entre os valores médios da AACPD total com a produtividade média. Obteve-se, ainda, a equação da curva ajustada aos valores da produtividade média dos grupos de cultivares em função da AACPD total, para o intervalo de severidade de doenças em estudo. 


\section{RESULTADOS E DISCUSSÃO}

Ocorreu elevada intensidade das manchas de Phaeosphaeria e de Cercospora no ensaio em Capão Bonito (Tabela 1). As duas doenças foram favorecidas pelas condições ambientais que ocorreram durante o período de condução do ensaio, onde a precipitação pluvial foi bem distribuída, com média mensal de $55,5 \mathrm{~mm}$ e de seis dias de chuva. A temperatura média variou de 14,9 a $25,7^{\circ} \mathrm{C}$ (média de $20,3^{\circ} \mathrm{C}$ ). Estas condições climáticas propiciaram umidade relativa do ar elevada, com formação de orvalho, durante praticamente todo o ciclo da cultura. Os demais ensaios da rede regional apresentaram produtividade baixa e severidade de doenças muito baixa em função da seca que ocorreu nas outras regiões do Estado de São Paulo.

A análise de variância dos dados de Capão Bonito para a produção de milho do ensaio deu os resultados da Tabela 2, onde se indica por três asteriscos a significância ao nível de $0,1 \%$ de probabilidade.

Também for am analisados os dados relativos a manchas de Cercospora (Tabela 3), de Phaeosphaeria (Tabela 4), com a mesma notação. Por sua vez, a Tabela 5 se refere à análise da variância referente a AACPD Total (manchas de Cercospora e da Phaeosphaeria).

Nos resultados obtidos em Capão Bonito, foi encontrada correlação entre a severidade das duas doenças, sendo o valor de $r=0,48 * * *$ (onde $\mathbf{r}$ é o coeficiente de correlação fenotípica, significativo a $0,01 \%$ pelo teste t). entre a AACPD de uma doença em relação à da outra, indicando relação entre a resistência dos híbridos a uma e à outra doença, o que permitiu constatar a influência delas na produtividade mesmo sob elevada severidade de ambas no ensaio.

Houve correlação negativa entre doenças e produtividade, observando-se, em média, menor produtividade dos híbridos quanto maior a severidade das doenças (Figuras 1 e 2). Analisando-se a severidade dos híbridos e suas respectivas produtividades, a correlação negativa mais alta foi obtida entre a área abaixo da curva de progresso da doença (AACPD) total e a produtividade, sendo o valor de $r=-0,76^{* * *}(\mathrm{P}<0,001)$. Por outro lado, os menores valores de correlação foram encontrados nas 
Tabela 1. Severidade das manchas de Phaeosphaeria e de Cercospora e produtividade de híbridos de milho (hibridos simples ou triplos) em ensaio instalado em Capão Bonito-SP, na safrinha 2002.

\begin{tabular}{|c|c|c|c|c|c|c|}
\hline \multirow{3}{*}{ Cultivares } & \multicolumn{5}{|c|}{ Severidade ${ }^{l}$} & \multirow{3}{*}{$\begin{array}{l}\text { Produti- } \\
\text { vidade } \\
\text { (kg/ha) }\end{array}$} \\
\hline & \multicolumn{2}{|c|}{$\begin{array}{c}\text { Mancha de } \\
\text { Phaeosphaeria }\end{array}$} & \multicolumn{2}{|c|}{$\begin{array}{l}\text { Mancha de } \\
\text { Cercospora }\end{array}$} & \multirow{2}{*}{$\begin{array}{l}\mathrm{AACPD}^{3} \\
\text { total }\end{array}$} & \\
\hline & Notas $^{2}$ & $\mathrm{AACPD}^{3}$ & $\operatorname{Notas}^{2}$ & $\mathrm{AACPD}^{3}$ & & \\
\hline A 2560 & $2,0 \mathrm{e}$ & $55,3 \mathrm{e}$ & $2,3 \mathrm{~d}$ & $57,4 \mathrm{~d}$ & $112,8 \mathrm{f}$ & $6510 \mathrm{a}$ \\
\hline Strike & $2,6 \mathrm{e}$ & $66,8 \mathrm{e}$ & $2,3 \mathrm{~d}$ & $57,5 \mathrm{~d}$ & $124,3 \mathrm{f}$ & $5507 \mathrm{c}$ \\
\hline DKB 333B & $3,5 \mathrm{~d}$ & $86,1 \mathrm{c}$ & $2,2 \mathrm{~d}$ & $51,5 \mathrm{~d}$ & $137,6 \mathrm{f}$ & $6515 \mathrm{a}$ \\
\hline A 2555 & $3,7 \mathrm{c}$ & $98,3 \mathrm{c}$ & $2,7 \mathrm{~d}$ & $60,7 \mathrm{~d}$ & $159,0 \mathrm{e}$ & $5065 \mathrm{~d}$ \\
\hline Fort & $2,5 \mathrm{e}$ & $65,2 \mathrm{e}$ & $3,5 \mathrm{c}$ & $94,5 \mathrm{~b}$ & $159,7 \mathrm{e}$ & 5929 b \\
\hline XB 7012 & $3,7 \mathrm{c}$ & $96,4 \mathrm{c}$ & $2,8 \mathrm{~d}$ & $68,8 \mathrm{~d}$ & $165,2 \mathrm{e}$ & $5232 \mathrm{c}$ \\
\hline XGN 01101 & $3,2 \mathrm{~d}$ & $79,7 \mathrm{~d}$ & $3,3 \mathrm{c}$ & $88,1 \mathrm{c}$ & $167,8 \mathrm{e}$ & $5135 \mathrm{~d}$ \\
\hline AG 7575 & $3,2 \mathrm{~d}$ & $68,5 \mathrm{e}$ & $3,7 \mathrm{c}$ & $100,3 \mathrm{~b}$ & $168,4 \mathrm{e}$ & $4700 \mathrm{~d}$ \\
\hline Valent & $3,3 d$ & $80,8 d$ & $3,5 \mathrm{c}$ & $90,0 \mathrm{c}$ & $170,7 \mathrm{e}$ & $5868 \mathrm{~b}$ \\
\hline DKB 350 & $3,5 \mathrm{~d}$ & $91,1 \mathrm{c}$ & $3,0 \mathrm{c}$ & $81,6 \mathrm{c}$ & $172,7 \mathrm{e}$ & $5417 \mathrm{c}$ \\
\hline $30 \mathrm{~K} 75$ & $3,3 \mathrm{~d}$ & $75,8 \mathrm{~d}$ & $3,8 \mathrm{c}$ & $97,6 \mathrm{~b}$ & $173,4 \mathrm{e}$ & $5551 \mathrm{c}$ \\
\hline AG 5011 & $4,0 \mathrm{c}$ & $95,6 \mathrm{c}$ & $3,3 \mathrm{c}$ & $86,5 \mathrm{c}$ & $182,1 \mathrm{~d}$ & $4679 \mathrm{~d}$ \\
\hline BRS 3060 & $4,2 \mathrm{c}$ & $112,8 \mathrm{c}$ & $3,0 \mathrm{c}$ & $73,5 \mathrm{c}$ & $186,3 \mathrm{~d}$ & $5034 \mathrm{~d}$ \\
\hline AS3466Top & $4,2 \mathrm{c}$ & $98,0 \mathrm{c}$ & $3,5 \mathrm{c}$ & $91,1 \mathrm{c}$ & $189,1 \mathrm{~d}$ & $4465 \mathrm{e}$ \\
\hline DAS 9560 & $3,7 \mathrm{c}$ & $91,8 \mathrm{c}$ & $4,3 \mathrm{~b}$ & $101,0 \mathrm{~b}$ & $192,8 d$ & $4939 \mathrm{~d}$ \\
\hline XB 7011 & $4,0 \mathrm{c}$ & $112,0 \mathrm{c}$ & $3,2 \mathrm{c}$ & $84,7 \mathrm{c}$ & $196,7 \mathrm{~d}$ & $4788 \mathrm{~d}$ \\
\hline Tork & $3,8 \mathrm{c}$ & $102,2 \mathrm{c}$ & $4,5 \mathrm{~b}$ & $102,1 \mathrm{~b}$ & $204,3 \mathrm{c}$ & $5328 \mathrm{c}$ \\
\hline BRS 3151 & $5,2 b$ & $132,2 \mathrm{~b}$ & $3,2 \mathrm{c}$ & $74,3 \mathrm{c}$ & $206,5 \mathrm{c}$ & $4664 d$ \\
\hline $30 \mathrm{~F} 90$ & $4,0 \mathrm{c}$ & $98,7 \mathrm{c}$ & $4,3 \mathrm{~b}$ & 107,8 b & $206,6 \mathrm{c}$ & $5833 \mathrm{~b}$ \\
\hline DAS 301 & $4,5 \mathrm{c}$ & $103,2 \mathrm{c}$ & $3,7 \mathrm{c}$ & $104,5 \mathrm{~b}$ & $207,7 \mathrm{c}$ & $4484 \mathrm{e}$ \\
\hline AGN32M43 & $4,0 \mathrm{c}$ & $96,0 \mathrm{c}$ & $4,3 \mathrm{~b}$ & $111,7 \mathrm{~b}$ & $207,7 \mathrm{c}$ & $4548 \mathrm{~d}$ \\
\hline CD 305 & $3,7 \mathrm{c}$ & $94,5 \mathrm{c}$ & $4,7 \mathrm{~b}$ & $119,3 \mathrm{a}$ & $213,7 \mathrm{c}$ & $4297 \mathrm{e}$ \\
\hline Exceler & $4,7 \mathrm{c}$ & $104,7 \mathrm{c}$ & $4,7 \mathbf{b}$ & $109,7 \mathrm{~b}$ & $214,4 \mathrm{c}$ & $4918 d$ \\
\hline DAS 8480 & $4,5 \mathrm{c}$ & $104,4 c$ & $4,2 \mathrm{~b}$ & $112,5 \mathrm{~b}$ & $216,8 \mathrm{c}$ & $5456 \mathrm{c}$ \\
\hline DKB 747 & $4,3 \mathrm{c}$ & $106,3 \mathrm{c}$ & $4,7 \mathbf{b}$ & $112,0 \mathrm{~b}$ & $218,3 \mathrm{c}$ & $4566 d$ \\
\hline AGN 3180 & $4,5 \mathrm{c}$ & $113,9 \mathrm{c}$ & $4,5 \mathbf{b}$ & $106,7 \mathrm{~b}$ & $220,7 c$ & $4719 \mathrm{~d}$ \\
\hline $30 \mathrm{~F} 98$ & $4,0 \mathrm{c}$ & $89,2 \mathrm{c}$ & $5,7 \mathrm{a}$ & 135,3 a & $224,4 \mathrm{~b}$ & $5078 \mathrm{~d}$ \\
\hline DAS 2 C599 & $4,5 \mathrm{c}$ & $109,0 \mathrm{c}$ & $4,3 \mathrm{~b}$ & $116,3 \mathrm{~b}$ & $225,3 \mathrm{~b}$ & $4589 \mathrm{~d}$ \\
\hline DAS 8550 & $4,5 \mathrm{c}$ & $121,6 \mathrm{~b}$ & $4,3 \mathrm{~b}$ & $107,1 b$ & $228,6 \mathrm{~b}$ & $4677 d$ \\
\hline AS 1533 & $4,0 \mathrm{c}$ & $103,7 \mathrm{c}$ & $5,2 \mathrm{a}$ & 126,9 a & $230,6 \mathrm{~b}$ & $4741 \mathrm{~d}$ \\
\hline
\end{tabular}


Tabela 1. continuação

\begin{tabular}{lcccccc}
\hline Master & $4,7 \mathrm{c}$ & $110,4 \mathrm{c}$ & $5,0 \mathrm{a}$ & $124,7 \mathrm{a}$ & $235,1 \mathrm{~b}$ & $4715 \mathrm{~d}$ \\
XB 7288 & $5,5 \mathrm{~b}$ & $134,8 \mathrm{~b}$ & $3,8 \mathrm{c}$ & $100,2 \mathrm{~b}$ & $235,1 \mathrm{~b}$ & $4811 \mathrm{~d}$ \\
DAS 8330 & $4,2 \mathrm{c}$ & $104,4 \mathrm{c}$ & $5,0 \mathrm{a}$ & $131,5 \mathrm{a}$ & $235,9 \mathrm{~b}$ & $5139 \mathrm{~d}$ \\
AL 30 & $4,3 \mathrm{c}$ & $101,7 \mathrm{c}$ & $5,3 \mathrm{a}$ & $135,3 \mathrm{a}$ & $237,0 \mathrm{~b}$ & $4129 \mathrm{e}$ \\
DAS 766 & $5,8 \mathrm{~b}$ & $130,6 \mathrm{~b}$ & $4,0 \mathrm{~b}$ & $110,6 \mathrm{~b}$ & $241,2 \mathrm{~b}$ & $4636 \mathrm{~d}$ \\
CD 304 & $5,7 \mathrm{~b}$ & $126,9 \mathrm{~b}$ & $5,3 \mathrm{a}$ & $140,3 \mathrm{a}$ & $267,2 \mathrm{a}$ & $4347 \mathrm{e}$ \\
AGN 3150 & $6,3 \mathrm{a}$ & $155,1 \mathrm{a}$ & $4,3 \mathrm{~b}$ & $114,0 \mathrm{~b}$ & $269,1 \mathrm{a}$ & $4153 \mathrm{e}$ \\
Speed & $5,7 \mathrm{~b}$ & $129,6 \mathrm{~b}$ & $5,7 \mathrm{a}$ & $140,7 \mathrm{a}$ & $270,2 \mathrm{a}$ & $4621 \mathrm{~d}$ \\
AS 3430 & $6,7 \mathrm{a}$ & $152,4 \mathrm{a}$ & $4,5 \mathrm{~b}$ & $121,6 \mathrm{a}$ & $274,0 \mathrm{a}$ & $4504 \mathrm{e}$ \\
XGN 01206 & $6,7 \mathrm{a}$ & $155,4 \mathrm{a}$ & $4,8 \mathrm{a}$ & $118,9 \mathrm{a}$ & $274,3 \mathrm{a}$ & $4042 \mathrm{e}$ \\
SHS 5070 & $6,8 \mathrm{a}$ & $177,6 \mathrm{a}$ & $5,0 \mathrm{a}$ & $120,0 \mathrm{a}$ & $297,6 \mathrm{a}$ & $4268 \mathrm{e}$ \\
\hline Média & 4,32 & 106,0 & 4,05 & 102,4 & 208,4 & 4921 \\
CV & $5,58 \%$ & $5,59 \%$ & $5,12 \%$ & $5,03 \%$ & $7,20 \%$ & $6,63 \%$ \\
\hline
\end{tabular}

1. Médias seguidas por letras distintas na mesma coluna estão agrupadas em diferentes classes que diferem entre si pelo teste de Scott-Knott a 5\% de significância (Análise dos dados ( $\mathbf{x}$ ) de severidade de cada doença transformados em ).

2. Notas de 1 a 9 correspondendo, respectivamente, às severidades de $0 ; 1 ; 2,5 ; 5 ; 10$; $25 ; 50 ; 75$ e mais de $75 \%$ de área foliar afetada. Quarta avaliação, realizada aos 115 dias após a semeadura.

3. $\mathrm{AACPD}=$ área abaixo da curva de progresso da doença.

Tabela 2. Análise da produção de milho do experimento de Capão Bonito, SP.

\begin{tabular}{lrrc}
\hline Causas da Variação & G.L. & Q.M. & F \\
\hline Blocos & 2 & 1.473 .579 & $13,86^{* * * * *}$ \\
Tratamentos & 41 & 1.086 .812 & $10,22^{* * * *}$ \\
Resíduo & 82 & 106.301 & \\
\hline
\end{tabular}

Coeficiente de variação $\mathrm{CV}=6,63 \%$. 
Tabela 3. Análise da variância relativa a manchas de Cercospora.

\begin{tabular}{lrcc}
\hline Causas da Variação & G.L. & Q.M. & \multicolumn{1}{c}{ F } \\
\hline Blocos & 2 & 0,6547 & 2,57 NS \\
Tratamentos & 41 & 4,2765 & $16,79 * * *$ \\
Resíduo & 82 & 0,2547 & \\
\hline
\end{tabular}

Coeficiente de variação $\mathrm{CV}=5,03 \%$. NS = Não significativa ao nível de $5 \%$.

Tabela 4. Análise da variância relativa a manchas de Phaeosphaeria.

\begin{tabular}{lrcc}
\hline Causas da Variação & G.L. & Q.M. & F \\
\hline Blocos & 2 & 2,0910 & $6,41^{* *}$ \\
Tratamentos & 41 & 4,5661 & $14,01^{* * *}$ \\
Resíduo & 82 & 0,3260 & \\
\hline
\end{tabular}

Coeficiente de variação $\mathrm{CV}=5,59 \%$.

Tabela 5. Análise da variância relativa a AACPD Total (manchas de Cercospora e de Phaeosphaeria).

\begin{tabular}{lrrr}
\hline Causas da Variação & G.L. & Q.M. & \multicolumn{1}{c}{ F } \\
\hline Blocos & 2 & $2.050,06$ & $9,11 * * *$ \\
Tratamentos & 41 & $5.203,11$ & $23,13 * * *$ \\
Resíduo & 82 & 224,91 & \\
\hline
\end{tabular}

Coeficiente de variação $\mathrm{CV}=7,20 \%$.

primeiras épocas de avaliação, aos 83 e 92 dias após a semeadura, para cada uma das doenças com a produtividade. Os valores de $\mathbf{r}$ em cada uma das quatro épocas para a mancha de Phaeosphaeria foram $-0,57 * * *$. $-0,64 * * ;-0,65 * * *$ e $-0,70 * * *$ e para a mancha de Cercospora, $-0,55 * * *$; $-0,56 * * * ;-0,61 * * *$ e $-0,59 * * *$, respectivamente.

Os valores das correlações entre a AACPD de cada uma das doenças com a produtividade foram de $-0,69 * * *$ e $-0,61^{* * *}$ para as manchas de 
Phaeosphaeria e de Cercospora, respectivamente, muito próximos aos das melhores avaliações tardias, mostram que as AACPDs também são adequadas para avaliar a influência de cada uma das doenças na produtividade.

Quanto à melhor época de avaliação de doenças, maiores valores de coeficiente de correlação foram obtidos na última avaliação, aos 115 dias após a semeadura para mancha de Phaeosphaeria e aos 106 e 115 dias, para a mancha de Cercospora. Estas épocas contrastam com a da safra normal, em que a avaliação mais adequada é realizada aproximadamente aos 90 dias após a semeadura, época em que no verão as plantas se encontram no final do estádio de grãos pastosos. Este fato pode ser explicado pelo atraso de até um mês no ciclo das plantas, ocasionado pela redução da temperatura no período de outono-inverno. Este atraso é bastante acentuado, principalmente no início da fase reprodutiva das plantas, retardando a maturação dos grãos, os quais levam mais tempo para atingir o estádio pastoso (Quiessi et al., 1999; Fancelli, 2001).

O maior valor do coeficiente de correlação da severidade da mancha de Phaeosphaeria com a produtividade em época mais tardia que a mancha de Cercospora pode ser explicado pelas diferenças relacionadas ao período de incubação dos dois patógenos. Bekman \& Paine (1982) observaram, em condições de casa-de-vegetação, a formação das primeiras lesões de C. zeae-maydis aos 9 dias após a inoculação e Fantin \& Balmer (1997) observaram, também em condições de casa-de-vegetação, o surgimento das primeiras lesões causadas por $P$. maydis apenas aos 26 dias após a inoculação. Ainda Godoy et al. (2000) estudando alterações na fotossintese e transpiração em folhas de milho infectadas por $P$. maydis, encontraram redução da eficiência fotossintética não apenas no tecido lesionado, mas também em parte do tecido verde remanescente das follhas infectadas. Observaram redução de $40 \%$ na taxa líquida de fotossíntese em folhas com severidade de doença de 10 a $20 \%$. Em vista destes dados, o maior valor de correlação da severidade de $P$. maydis em época mais tardia pode ter ocorrido em função da presença ainda assintomática do fungo, por um período mais longo, nos tecidos da planta.

De acordo com o teste de Scott-Knott $(\mathrm{P}<0,05)$, os híbridos foram agrupados, quanto à resistência à mancha de Phaeosphaeria, utili- 
zando a AACPD, em cinco classes, a saber: resistentes (R): A 2560, Fort, Strike e AG 7575; moderadamente resistentes (MR): 30K75, XGN01101 e Valent; intermediários (I): DKB 333B, 30F98, DKB 350, DAS 9560, CD 305, AG 5011, AGN 32M43, XB 7012, AS 3466 Top, A2555, 30F90, AL 30, Tork, DAS 301, AS1533, DAS 8480, DAS 8330, Exceler, DKB 747, DAS 2C599, Master, XB 7011, BRS 3060 e AGN 3180; moderadamente suscetiveis (MS): DAS 32, DAS 8550, CD 304, Speed, DAS 766, BRS 3151 e XB 7288 e suscetíveis (S): AS 3430, AGN 3150, XGN 01206 e SHS 5070. Os valores médios de severidade da mancha de Phaeosphaeria em cada classe, nas diferentes épocas de avaliação, estão apresentados na Figura 1.

Quanto à mancha de Cercospora, o agrupamento resultou em quatro classes: resistentes (R): DKB 333B, A 2560, Strike, A 2525 e XB 7012; moderadamente resistentes (MR): BRS 3060, BRS 3151; DKB 350, XB 7011, AG 5011, XGN 01101, Valent e AS 3466 Top; moderadamente suscetiveis (MS): Fort, 30K75, XB 7288, AG 7575, DAS 9560, Tork, DAS 301, AGN 3180, DAS 8550, 30F90, Exceler, DAS 766, AGN 32M43, DKB 747, DAS 32, DAS 8480, AGN 3150 e DAS 2C599 e suscetiveis (S): XGN 01206, CD 305, SHS 5070, AS 3430 , Master, AS 1533, DAS 8330, 30F98, AL 30, CD 304 e Speed. Os valores médios de severidade da mancha de Cercospora em cada classe, nas diferentes épocas de avaliação, estão apresentados na Figura 2.

Ainda, de acordo com a produtividade, os híbridos puderam ser agrupados em 5 classes, sendo os mais produtivos DKB 333B e A 2560 seguidos por Fort, Valent e 30F90 e estes por 30K75, Strike, DAS 8480 , DKB 350, Tork e XB 7012. Grande parte deles apresentou produtividade intermediária: DAS 8330, XGN 01101, 30F98, A 2555, BRS 3060 , DAS 9560, Exceler, XB 7288, XB 7011, AS 1533, AGN 3180, Master, AG 7575, AG 5011, DAS 8550, BRS 3151, DAS 766, Speed, DAS 2C599, DKB 747 e AGN 32M43 enquanto os de menor produtividade foram: AS 3430, DAS 301, AS 3466 Top, CD 304, CD 305, SHS 5070, AGN 3150, AL 30, DAS 32 e XGN 01206 (Tabela 1).

O efeito de decréscimos na produtividade decorrentes de severidades crescentes da mancha de Phaeosphaeria, encontrado neste traba- 


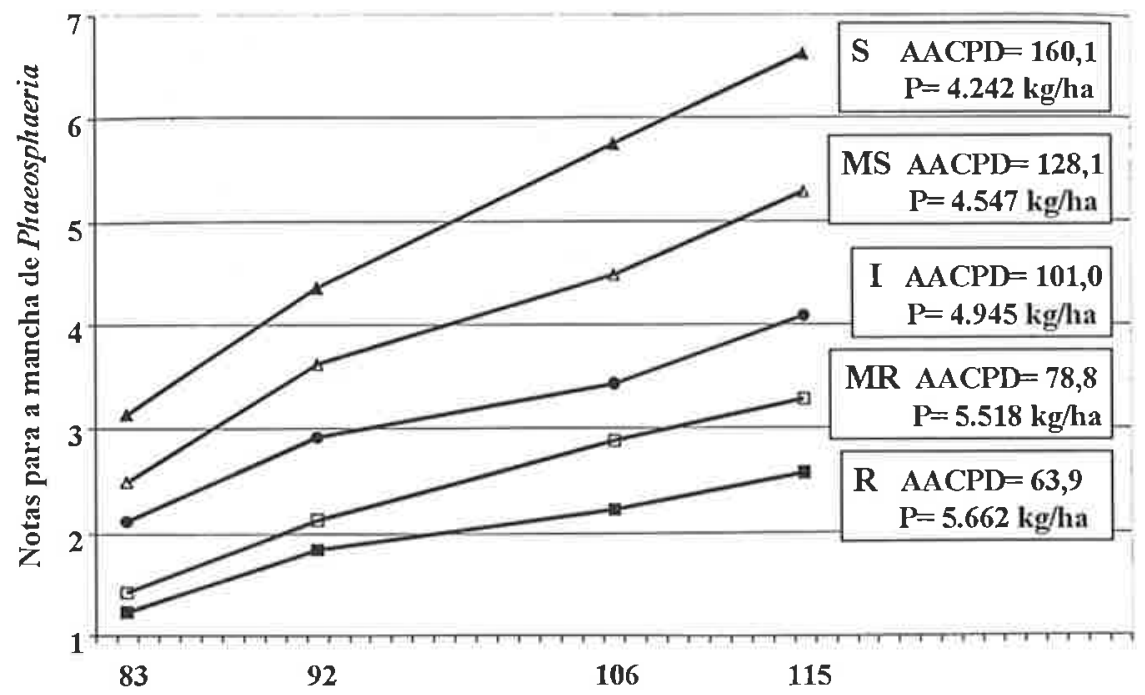

Dias após a semeadura

Figura 1. Severidade da mancha de Phaeosphaeria (notas de 1 a 9 correspondendo, respectivamente, às severidades de $0 ; 1$; 2,$5 ; 5 ; 10 ; 25 ; 50 ; 75$ e mais de $75 \%$ de área foliar afetada) em 4 épocas de avaliação e respectivos valores médios da área sob a curva de progresso da doença (AACPD) e de produtividade $(\mathrm{P})$ de grupos de híbridos de milho que se comportaram como resistentes $(\mathrm{R})$, moderadamente resistentes (MR), intermediários (I), moderadamente suscetíveis (MS) e suscetíveis (S) na safrinha 2002 em Capão Bonito-SP.

1ho, também foi observado por Sawazaki et al. (1997), Brasil \& Carvatho (1998), Pacheco et al. (1999) e Santos et al. (2002) em estudos realizados com outros híbridos.

Apesar da correlação negativa observada entre quantidade de doenças e produtividade encontradas no atual estudo, alguns hibridos, como 30F90 e DAS 8480 tiveram elevada produtividade, embora apresentando moderada porcentagem de área foliar coberta por manchas. Estudos 


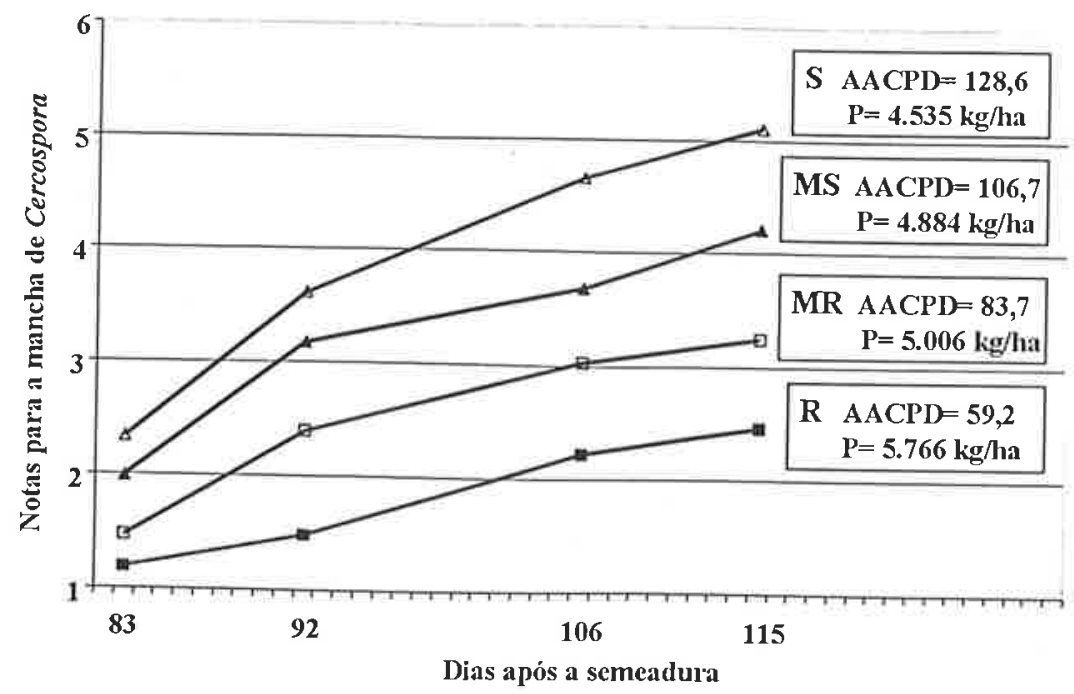

Figura 2. Severidade da mancha de Cercospora (notas de 1 a 9 correspondendo, respectivamente, às severidades de $0 ; 1 ; 2,5 ; 5$; 10; 25; 50; 75 e mais de $75 \%$ de área foliar afetada) em 4 épocas de avaliação e respectivos valores médios da área sob a curva de progresso da doença (AACPD) e de produtividade (P) de grupos de híbridos de milho que se comportaram como resistentes (R), moderadamente resistentes (MR), moderadamente suscetiveis (MS) e suscetíveis (S) na safrinha 2002 em Capão Bonito-SP.

realizados por Von Pinho et al. (2002), em Lavras-MG sobre danos causados pela mancha de Phaeosphaeria em oito cultivares de milho, mostraram resultados semelhantes com o cultivar 30F33, que não apresentou diminuição do rendimento de grãos apesar da alta severidade da doença, em contraste com os demais cultivares em estudo. Menten et al. (1996), em ensaio conduzido em Espírito Santo do Pinhal-SP com nove cultivares de milho, sob elevada intensidade da mancha de Phaeosphaeria, embora tenha encontrado dois cultivares mais produtivos e com severidade moderada da doença, observou divergências quanto à resposta da resistência de alguns híbridos, comparados aos resultados obtidos em traba- 
Ihos de outros autores e também diferenças de potencial produtivo entre os híbridos estudados, dificultando a observação do efeito da doença sobre a produtividade. Ainda Miranda et al. (2002) estudando a reação de 25 hibridos de milho em ambientes com alto e baixo teor de nitrogênio (N), em Coimbra-MG, observaram maior severidade média da doença sob o nivel mais alto de $\mathrm{N}$ e também que alguns genótipos apresentaram interação com o ambiente, comportando-se como mais resistentes ou como mais suscetiveis em relação aos demais em função da maior adubação nitrogenada em cobertura.

Estas variações de resultados reforçam a necessidade, já citada por Fantin et al. (1990), de avaliar o comportamento de materiais genéticos em diferentes regiões ao visar à indicação para cultivo. Além disso, Fantin et al. (1999) e Paterniani et al. (2000) alertam para a necessidade de verificar a estabilidade e durabilidade da resistência exibida por genótipos de milho.

Para estudar a influência da severidade das duas doenças conjuntamente sobre a produtividade média, o agrupamento dos híbridos feito através do teste de Scott-Knott a 5\%, com base na AACPD total, permitiu a formação de 6 classes (Tabela 1), onde os híbridos apresentaram severidade das doenças variando de muito baixa: A 2560, Strike e DKB 333B; baixa: A 2555, Fort, XB 7012, XGN 01101, AG 7575, Valent, DKB 350 e 30K75; baixa a moderada: AG 5011, BRS 3060, AS 3466 Top, DAS 9560 e XB 7011; moderada: Tork, BRS 3151, 30F90, DAS 301, AGN 32M43, CD 305, Exceler, DAS 8480, DKB 747 e AGN 3180; moderada a alta: DAS 2C599, DAS 8550, AS 1533, DAS 32, Master, XB 7288, DAS 8330, AL 30 e DAS 766 e alta: CD 304, AGN 3150, Speed, AS 3430, XGN 01206 e SHS 5070. Obteve-se elevado valor da correlação fenotípica $\left(r=-0,94^{* *}\right)$ das médias de classes desta variável com a produtividade, pela redução do efeito de dispersão causado pelas diferenças de potencial produtivo dos híbridos.

Obteve-se uma equação polinomial de segundo grau ajustada aos valores da produtividade em função da AACPD total (Figura 3), que revelou redução na produtividade média dos híbridos a partir de niveis muito baixos de severidade de doenças, ao redor de $\mathrm{AACPD}=125$, o 
que equivaleu a severidades, na última avaliação, inferiores à nota $3(2,5 \%$ de área foliar com sintomas) para cada uma das doenças (Figuras 1, 2 e 3). Sawazaki et al. (1997) em estudo realizado em três regiões do Estado de São Paulo obtiveram resultados semelhantes em relação à mancha de Phaeosphaeria, encontrando maiores valores de correlações da severidade da doença com a produtividade com notas médias a partir de 3,5 (entre 2,5 e $5 \%$ de área foliar com sintomas). Por outro lado, Brasil \& Carvalho (1998), em estudos conduzidos em Goianésia-GO, somente detectaram reduções na produtividade sob intensidade mais elevada da doença.

No presente trabalho também se observou que, com o aumento dos valores da AACPD total até próximos de 275 , onde as notas médias atribuídas às manchas de Phaeosphaeria e de Cercospora foram ao redor de 6 ou mais ( $25 \%$ ou mais) e próximas a $5(10 \%$ de área foliar com sintomas), respectivamente, houve redução próxima a $2.000 \mathrm{~kg} / \mathrm{ha}$ na produtividade média dos híbridos (Figura 3). Observou-se também, no intervalo de severidade de doenças estudado, que incrementos nas notas com menores valores de severidade causaram maiores decréscimos na produtividade que os mesmos incrementos nos valores mais elevados da severidade, evidenciando a importância das doenças em níveis baixos de severidade (Figura 3).

Os resultados obtidos no presente trabalho permitiram concluir que avaliações tardias de doenças durante o cultivo da safrinha, com as plantas no final do estádio de grão pastoso, proporcionaram melhor correlação com a produtividade, sendo adequadas e suficientes para discriminar a resistência entre os genótipos de milho às doenças estudadas. Concluiu-se, também, que o aumento da intensidade das manchas de Phaeosphaeria e de Cercospora reduziu a produtividade de grãos do milho a partir de níveis muito baixos de severidade, inferiores a $2,5 \%$ de área foliar com sintomas de cada uma das doenças. 


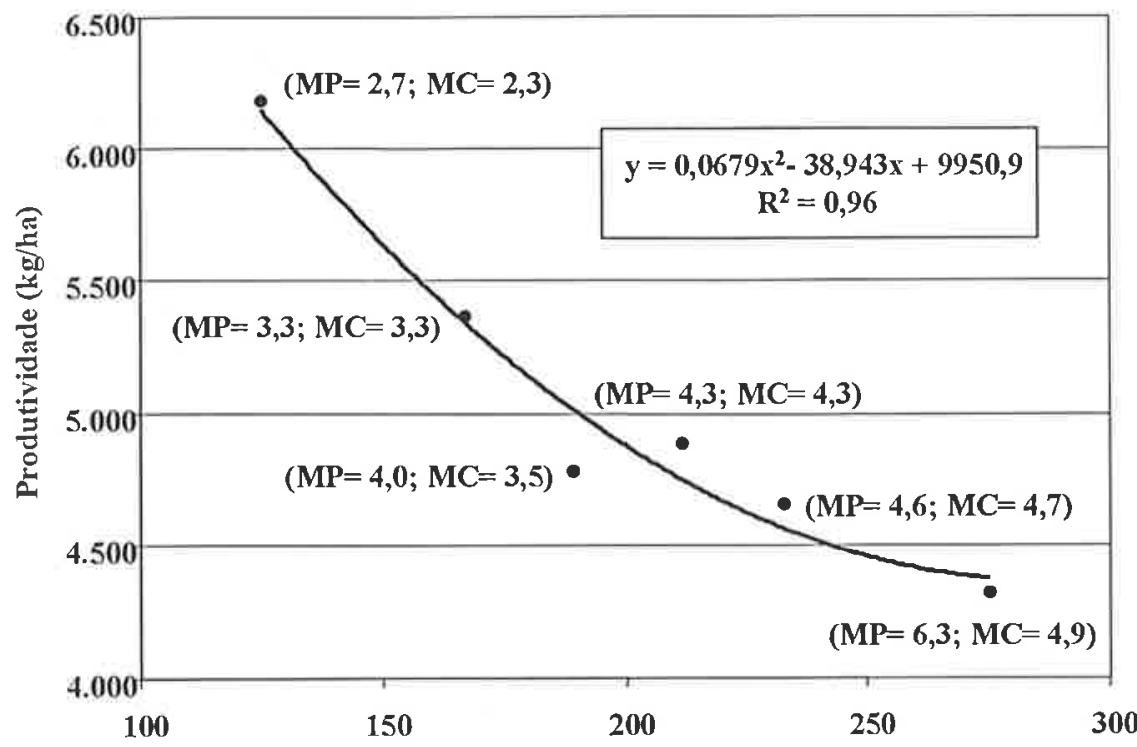

AACPD das manchas de Phaeosphaeria e de Cercospora

Figura 3. Produtividade média de híbridos de milho em função da severidade total de doenças, medida através dos valores médios da área abaixo da curva de progresso da doença (AACPD) total de 6 classes de híbridos, e respectivos valores médios de notas de severidade da mancha de Phaeosphaeria (MP) e da mancha de Cercospora (MC) na safrinha 2002 em Capão Bonito-SP.

\section{REFERÊNCIAS BIBLIOGRÁFICAS}

AGROCERES, 1993. Guia Agroceres de Sanidade. São Paulo, 56p. BECKMAN, P.M. \& G.A. PAYNE, 1982. External Growth, Penetration e Development of Cercospora zeae-maydis in Corn Leaves. Phytopathology, 72:810-815.

BRASIL, E.M. \& Y. CARVALHO, 1998. Comportamento de Híbridos de Milho em Relação a Phaeosphaeria maydis em Diferentes Épocas de Plantio. Pesquisa Agropecuária Brasileira, 33:1977-1981. 
CANTERI, M.G.; R.A. ALTHAUS; J.S. VIRGENS FILHO; E.A. GIGLIOTI; C.V. GODOY, 2001. SASM-Agri Sistema para Análise e Separação de Médias em Experimentos Agrícolas pelos Métodos Scott-Knott, Tukey e Duncan. Revista Brasileira de Agrocomputação, 1:18-24.

FANCELLI, A.L., 2001. Fisiologia das Plantas de Milho em Condições de Safrinha, In: SEMINÁRIO NACIONAL DE MILHO SAFRINHA, 6., Londrina, 2001. A Cultura do Milho Safrinha. Londrina: IAPAR. p.11-31.

FANTIN, G.M. 2004a. Avanço da Cercóspora. Cultivar - Grandes Culturas, 6:28-31.

FANTIN, G.M. \& E. BALMER, 1997. Método de Inoculação e Evolução de Sintomas da Mancha Foliar de Phaeosphaeria maydis em Milho. Summa Phytopathologica, 23:64-65. Suplemento.

FANTIN, G.M., 1990/1994. Mancha de Phaeosphaeria, Doença do Milho que vem Aumentando sua Importância. Biológico, 56:39.

FANTIN, G.M.; C. DUDIENAS; A.P. DUARTE; M.E.A.G.Z. PATERNIANI; P.C. RECO; L. COICEV; A.P. ARAÚJO; D. BOLONHEZI, 2000. Ocorrência e Intensidade de Doenças em Híbridos de Milho no Estado de São Paulo - Safra 98/99. Summa Phytopathologica, 26:110. Suplemento.

FANTIN, G.M.; C. DUDIENAS; A.P. DUARTE; E. SAWAZAKI; J.L. CASTRO; D. BOLONHEZI, 2004b. Evolução da Mancha de Cercospora do Milho no Estado de São Paulo. Summa Phytopathologica, 30:99-100. Suplemento.

FANTIN, G.M.; C. DUDIENAS; A.P. DUARTE; G. CAZENTINI FILHO; P.C. RECO; L. COICEV; M.E.A.G.Z. PATERNIANI; N. BORTOLETO; J.L. CASTRO, 1999. Ocorrência e Intensidade de Doenças em Cultivares de Milho. In: DUARTE, A.P. \& PATERNIANI, M.E.A.G.Z. (Coords.) Cultivares de Milho no Estado de São Paulo: Resultados das Avaliações Regionais IAC/CATI/ Empresas 1998/99. Campinas: Instituto Agronômico. p.35-56. (Documentos IAC, 66) 
FANTIN, G.M.; E. SAWAZAKI; A.P. DUARTE; L.A. SAES; A. PETINELLI JR., 1990. Reação de Cultivares de Milho a Doenças em Diferentes Regiões do Estado de São Paulo. Arquivos do Instituto Biológico, 57:25. Suplemento.

FANTIN, G.M.; K.R. BRUNELLI; I.C. RESENDE; A.P. DUARTE, 2001. A Mancha de Cerçospora do Milho. Campinas: IAC. 19p. (Boletim Técnico, 192)

FERNANDES, F.T.; E. OLIVEIRA, 2000. A Mancha por Cercospora em Milho. Sete Lagoas: Embrapa. 1p. (Comunicado Técnico, 16) GODOY, C.V.; L. AMORIM; A. BERGAMIN FILHO, 2001. Alterações na Fotossintese e na Transpiração de Folhas de Milho Infetadas por Phaeosphaeria maydis. Fitopatologia Brasileira, 26:209-213.

MENTEN, J.O.M.; A.L. PARADELA; L.M.D. MARTINS; S.R.A ARANTES, 1996. Reação de Genótipos de Milho à Phaeosphaeria maydis e Efeito na Produtividade. Ecossistema, 21:72-74.

MIGUEL-WRUCK, D.S.; J.M.V. PAES; R.K. ZITO; J.A. SOUZA, 2002. Avaliação da Severidade de Phaeosphaeria sp. e Cercospora sp. em Cultivares de Milho no Ano Agrícola 2001/2002 (compact disc). In: CONGRESSO NACIONAL DE MILHO E SORGO, 24, Florianópolis, 2002. Sete Lagoas: ABMS/Embrapa Milho e Sorgo/ Epagri. $4 \mathrm{p}$.

MIRANDA, G.V.; R.R. FIDELIS; A. VAZ DE MELO; L.V. SOUZA; M.O. SOARES; L.R. OLIVEIRA, 2002. Reação de Cultivares de Milho à Mancha de Phaeosphaeria em Condições de Estresse de Baixo Nitrogênio (compact disc). In: CONGRESSO NACIONAL DE MILHO E SORGO, 24, Florianópolis, 2002. Sete Lagoas: ABMS/Embrapa Milho e Sorgo/Epagri. 5p.

PACHECO, A.C.; L.C. VIEIRA; D.R.GUIMARÃES; T. CANTON, 1999. Reação de Híbridos de Milho à Mancha Foliar de Phaeosphaeria. Agrop. Catarinense, 12:14-16.

PATERNIANI, M.E.A.G.Z.; E. SAWAZAKI; C. DUDIENAS; J.C.V.N.A. PEREIRA, 2000. Cruzamentos Dialélicos de Linhagens de Milho sob Condições de Mancha de Phaeosphaeria. Scientia Agrícola, 57:313-318. 
PEGORARO, D.G.; E. VACARO; C.N. NUSS; F.K. DAL SOGLIO; M.J.C.M. SERENO; J.F. BARBOSA NETO, 2001. Efeito de Épocas de Semeadura e Adubação na Mancha Foliar de Phaeosphaeria em Milho. Pesquisa Agropecuária Brasileira, 36:1037-1042. QUIESSI, J.A.; A.P. DUARTE; S.J. BICUDO; M.E.A.G.Z. PATERNIANI, 1999. Rendimento de Grãos e Características Fenológicas do Milho em Diferentes Épocas de Semeadura, em Tarumã (SP) In: SEMINÁRIO SOBRE A CULTURA DO MILHO SAFRINHA, 5. Barretos, 1999. Anais. Campinas, Instituto Agronômico, p.239. 247.

SANTOS, P.G; F.C. JULIATTI; A.L. BUIATTI; O.T. HAMAWAKI, 2002. Avaliação do Desempenho Agronômico de Híbridos de Milho em Uberlândia-MG. Pesquisa Agropecuária Brasileira, 37:597-602. SAWAZAKI, E.; C. DUDIENAS; M.E.A.G.Z. PATERNIANI; J.C.C. GALVÃO; J.L. CASTRO; J. PEREIRA, 1997. Reação de Cultivares de Milho à Mancha de Phaeosphaeria no Estado de São Paulo. Pesquisa Agropecuária Brasileira, 32:585-589.

SHURTLEFF, M.C. (Ed.), 1992. Compendium of Corn Diseases. 2.ed. St. Paul: American Phytopathological Press. 105p.

VON PINHO, R.G; A.G. STEOLA; M.R. GROSS; J.P. SOUZA, 2002.

Reação de Cultivares e Danos Causados pela Mancha Branca do Milho (compact disc). In: CONGRESSO NACIONAL DE MILHO E SORGO, 24, Florianópolis, 2002. Sete Lagoas: ABMS/Embrapa Milho e Sorgo/Epagri. 5p.

WARD, J.M.J.; E.L. STROMBERG; D.C. NOWELL; F.W. NUTTER JR. 1999, Gray Leaf Spot: a Disease of Global Importance in Maize Production. Plant Disease, 83:884-895.

WHITE, D.G. (Ed.), 2000. Compendium of Corn Diseases. 3.ed. St. Paul: American Phytopathological Society. 78p. 